\title{
Pesquisa Bibliográfica no Contexto Brasileiro sobre a Aplicação do Design Etnográfico como Forma de Potencializar Projetos de Design
}

\author{
Bibliographical Research in the Brazilian Context on the Application of \\ Ethnographic Design to Enhance Design Projects
}

\author{
Angélica Porto Cavalcanti de Souza ${ }^{1}$, Camille Santiago Caminha ${ }^{1}$, Fábio Campos ${ }^{2}$, Paulo Carneiro da Cunha \\ Filho $^{3}$ \\ ${ }^{1}$ Curso de Design, Universidade Federal de Pernambuco, Recife, Brasil \\ ${ }^{2}$ Demartamento de Informática, Universidade Federal de Pernambuco, Recife, Brasil \\ ${ }^{3}$ Departamento de Comunicação, Universidade Federal de Pernambuco, Recife, Brasil
}

Correspondência: Angélica Porto Cavalcanti de Souza, Universidade Federal de Pernambuco, Endereço: Av. Prof. Moraes Rego, 1235, Cidade Universitária, CEP.: 50.670-901 Recife, Brasil. Tel: 55 81 2126-7729. E-mail: aportocs@gmail.com

Recebido: 5 de outubro de 2018 Aceito: 30 de novembro de 2018 Publicado: 31 de dezembro de 2018

DOI: http://dx.doi.org/10.21714/1679-18272018v16Ed.p222-230

\section{Resumo}

Este trabalho tem como base uma pesquisa de referências bibliográficas em artigos acadêmicos brasileiros que utilizam a abordagem etnográfica junto a do design, como meio para potencializar a atividade de projeção de artefatos com foco na compreensão do público em um contexto verdadeiramente centrado no ser humano com intuito de gerar insights no processo de Design. O objetivo é verificar o estado da arte nacional e entender o que a literatura fala sobre o potencial da etnografia para a área de Design, além de apresentar indicativos para pesquisas futuras. Os resultados encontrados ressaltam a importância da etnografia nesse contexto e apresentam formas de contribuição dentro de pesquisas e trabalhos de Design.

Palavras-chave: Design, etnografia, antropologia, design participativo, potencial.

\begin{abstract}
This work is based on a research of bibliographical references in Brazilian academic articles which use the ethnographic approach together with Design, to enhance the activity of projecting artifacts with a focus on understanding the public in a context truly centered on the human being intending to generate insights in the Design process. The main goal is to verify the state of national art to understand what the literature speaks about the potential of ethnography for the Design area, besides presenting indicatives for future research. The results founded highlight the importance of ethnography in this context and present forms of contribution within research and design work.
\end{abstract}

Keywords: Design, ethnography, anthropology, participatory design, potencial.

\section{Esta obra está licenciada sob uma Licença Creative Commons Attribution 3.0.}

\section{Introdução}

A atividade de design evoluiu de maneira drástica desde seus primórdios. Passou por características de cunho técnico-artesanal (assim como vistos na escola de Bauhaus na década de 20, ensinados e aplicados em esquemas de oficinas por mestres que, segundo Droste (2014), representavam o núcleo do treinamento dos cursos oferecidos), alcançando campos de produção a níveis industriais e produções em grande escala advindas da evolução das tecnologias e revoluções industriais, criando grandes impactos sociais como a divisão intensiva de trabalho, a 
diminuição dos preços e a criação de novos paradigmas.

Após os adventos industriais, as novas formas de produção em escala também trouxeram historicamente novos problemas como os de natureza ecológica, além de novas preocupações. O sistema de produção, que tendia a valorizar pontos como as formas mais lucrativas de confecção para empresas ou os materiais a serem utilizados nessa produção, alcançou uma nova abordagem, a compreensão centrada nos usuários (necessidades, pontos de dor, rotinas, rituais, sentimentos).

É nesse entendimento, de seus costumes e comportamentos, colocando-o como elemento mais importante no campo de trabalho de um designer, que é possível perceber potencialidades para produção de artefatos que trazem verdadeiro significado e suprem de maneira eficaz e eficiente suas necessidades primordiais.

Um caminho que já é internacionalmente disseminado e aplicado para a compreensão do usuário, tanto na área acadêmica como no mercado, em grandes empresas, vem da interdisciplinaridade dos campos das ciências sociais, da antropologia e do design. Essa abordagem oferece um verdadeiro mergulho na realidade da vida dos usuários em diferentes contextos, de uma maneira focada e adaptada para as necessidades dos profissionais do campo de design, no intuito de potencializar as chances não apenas de melhorar produtos já existentes no mercado como também de criar, com base nas constantes evoluções tecnológicas, projetos que sejam verdadeiramente úteis, eficazes, eficientes e que tenham verdadeiro significado, consequentemente, embutido de real valor.

Considerando a importância e complexidade do processo criativo característico dos trabalhos dos designers, o conhecimento do usuário em um grau profundo proporciona grandes chances para insights.

No contexto internacional, a literatura traz a relação entre design e antropologia como duas áreas das ciências sociais que possuem potencial para congruência e criação de uma nova abordagem. Esses trabalhos são em sua maioria evidências aplicadas e abordam também temas de discussão em relação ao método etnográfico no Design e suas variações, além de relacionar esse campo com as áreas de IHC e tecnologia. Apesar de existente, segundo Mota e Sirito (2016) o Design Antropológico ainda é uma área relativamente nova no campo de pesquisa do design e ainda está em desenvolvimento. Para Pink (2014), o Design Antropológico possibilita um trabalho em um mundo temporal, onde o futuro está incluído (diferentemente da antropologia) e que procura intervir nesse futuro para produzir mudanças.

A professora Sarah Pink, doutora antropóloga, professora no Real Melbourne Institute of Technology na Austrália, é uma profissional altamente atuante no campo do Design Antropológico e suas contribuições seguem tanto sua participação como consultora para grandes empresas que visam a busca para caminhos de inovação em profissões de futuro, quanto em ricas contribuições acadêmicas, disseminando suas contribuições em nível teórico e empírico.

Para Pink (2013), design é uma atividade situada e construtiva de criação de significado e construção e, segundo Segelstrom e Holmlid (2015), a apropriação da etnografia é aplicada com o propósito de "informar o design". Pink corrobora com essa visão e que, o design ao invés de tentar mudar comportamentos, deve conhecer a relação da intervenção com as rotinas existentes (PINK; LEDER MACKLEY, 2013) e imergir de forma situada.

A antropologia oferece métodos, aplicados na imersão do contexto, que, de uma maneira mais eficiente, focado para objetivos específicos no interesse do campo do designer como projetista de inovação ou solucionador de problemas, resulta em ricos insights. Esses métodos de cunho qualitativo, por muitas vezes recebem rígidas críticas sobre o fato de ser uma abordagem muito subjetiva e produzir resultados dependentes do observador e suscetível à variáveis incontroláveis.

No entanto, Borman, LeCompte e Goetz (1986) defendem essa abordagem de pesquisa e rebatem críticas comumente feitas, porém, entendem que são pesquisas válidas apenas quando são muito bem-feitas. Esse fazer bem é caracterizado pelos autores como a capacidade do método de encontrar níveis de padrão, durante as recorrências na análise pelo caminho da triangulação do estado da arte juntamente com os dados coletados em pesquisa com os participantes.

Os métodos do campo da antropologia que são amplamente aplicados no contexto do design para compreensão dos comportamentos envolvem dos mais básicos e clássicos como observação, questionário, entrevista semiestruturada, focus group e mapeamentos aos mais recentes como video tour, video reenactment, lurking entre outros que são muitas vezes mais exploratórios.

Segundo Stappers et al. (2009), na década de 80, o focus group, testes de usabilidade e conceitos com o usuário em laboratório, se tornaram uma prática emergente no Design. Apesar disso, no campo de interdisciplinaridade, 
os designers adaptam as técnicas provindas da antropologia (SEGELSTRÖM; HOLMLID, 2015) apresentando os resultados de uma forma mais pragmática e rápida, criando métodos de observação e entrevistas mais focadas em seus objetivos, como entrevistas temáticas, fly on the wall, netnografia, behavioral mapping, entre outros.

Compreendendo que internacionalmente o campo do design etnográfico está consolidado e sua aplicação engloba campos tanto de estudos acadêmicos quanto aplicados empiricamente no mercado, o questionamento levantado para essa pesquisa é: como a etnografia é atualmente aplicada no contexto do design brasileiro?

Este trabalho visa verificar o estado da arte nacional que utiliza a abordagem etnográfica, junto ao design, comparados com a literatura internacional, a fim de entender como acontece essa abordagem e como é visto o potencial para as pesquisas de design no cenário nacional, além de apresentar indicativos para trabalhos futuros.

\section{Metodologia}

Objetivando a comparação sobre o conhecimento e a aplicação da abordagem etnográfica no Brasil e em diferentes países, como filtro de delimitação, em dezembro de 2017, foram pesquisados artigos publicados em periódicos e congressos brasileiros em contraste com os encontrados em periódicos e congressos internacionais. Tratando-se de uma pesquisa de caráter amplamente contextualizado, a citação das localidades nos textos dos artigos também atuou como filtro para delimitação dos grupos para pesquisa entre publicações nacionais e internacionais.

A pesquisa exploratória internacional foi realizada em anais e congressos, nas bases ACM e SAGE, utilizando as palavras-chave "anthropology", "ethnography" e "design" nos abstracts. A pesquisa nacional de cunho exploratório foi desenvolvida utilizando a ferramenta de pesquisa online específica para trabalhos acadêmicos, o Google Scholar no intuito de encontrar os trabalhos no contexto nacional, utilizando palavras chave "antropologia", "etnografia" e "design" no título e conteúdo das publicações. Outro critério adotado foi a coleta de artigos publicados no espectro temporal, entre 2008 e 2017, cujo tema ou resumo estivesse relacionado com design antropológico e/ou design etnográfico.

Para análise dos dados, os artigos selecionados foram lidos em sua totalidade e, posteriormente, foi criada uma tabela com os seguintes campos de análise destacando os achados em cada artigo selecionado:

- $\quad$ a abordagem, no intuito de entender os temas que são abordados envolvendo as palavras chaves pesquisadas e o tipo de análise empregada, isto é, de bases teóricas ou aplicadas;

- definição de Design Etnográfico, para analisar como as pesquisas brasileiras conceituam a abordagem do Design Etnográfico;

- definição de Design Antropológico, para analisar como as pesquisas brasileiras conceituam o campo do Design Antropológico, sendo esse um campo relativamente recente e ainda em desenvolvimento;

- potencial para o design, no objetivo de entender como esses artigos compreendem quais as potencialidades de estudar e/ou aplicar os métodos do Design Etnográfico e Design Antropológico;

- exemplos de estudos, identificando qual os temas e objetivos dos contextos e exemplos empíricos abordados nos textos; e

- técnicas utilizadas, para identificar qual a variedade das técnicas que são aplicadas ou citadas nos estudos.

\section{A Etnografia e a literatura do design nacional}

Como citado anteriormente, a pesquisa nas bases nacionais sobre artigos que envolvessem a abordagem de design antropológico e design etnográfico foi de caráter exploratório e resultou em 13 artigos nos últimos 10 anos, entre 2008 e 2017, sendo o mais antigo datado de 2011 e o mais recente de 2017.

Das bases encontradas 10 fazem parte de revistas ou congressos no campo de design (Arcos Design, P\&D, InfoDesign, Design Research Journal e Logo) e 3 em campos diversos, englobando áreas de "Ciências matemáticas e computação", "Literatura, linguística e artes" e uma revista sobre "Tecnologias para competitividade industrial".

\subsection{Abordagem dos artigos nacionais}

Os artigos brasileiros analisados, da mesma forma dos artigos internacionais, também tratam da relação entre Design e Antropologia, e abordam como método a etnografia junto ao design. A relação entre design e antropologia, denominada recentemente de Design Antropológico, já é aceita tanto na literatura nacional quanto na internacional. 
Sobre as temáticas tratadas nos artigos selecionados, os assuntos apresentam uma grande variedade de abordagens relacionadas aos campos e diferentes objetivos. Foram identificados artigos que tratam e aplicam a etnografia em espaços físicos (cidade do Rio de Janeiro, escritório de design) e espaços digitais (em netnografias e em IHC), arte (análises de livros fotográficos), inovação tecnológica e debates teóricos (sobre o estudo da etnografia rápida e as contribuições das ciências sociais para o campo do design). Essa grande diversidade valida uma notável flexibilidade que o campo do design antropológico pode oferecer para sua aplicação.

Outra questão que vale destacar é a presença maior da etnografia utilizada na exploração do problema e para compreender comportamentos e situações. A etnografia como método de design vem sendo utilizada em várias áreas de estudo, no entanto, com base no estado da arte pesquisado, percebe-se a restrição da literatura na utilização do método em outras etapas do processo de design além do reconhecimento inserido no contexto e compreensão dos usuários, como por exemplo nas fases de ideação, prototipação e testes.

Nos artigos selecionados também foi identificado a relação entre etnografia e design participativo, ainda que em apenas 2 das 13 publicações analisadas. Vale ressaltar que o processo de colaboração entre etnografia e o design proporciona grande contribuição no processo criativo. Vasconcelos (2012) fala que "o uso dos métodos na fase de exploração de problemas clarifica as ideias durante o processo de design, proporcionando um plano organizado de ação para apoiar a tarefa de projetar produtos". Dessa forma, o design etnográfico, ao proporcionar uma relação de colaboração entre o usuário e o designer em um contexto participativo, possui também o potencial para maior compreensão dos problemas e situações do usuário, ideias mais claras e geração de insights.

Apesar de tudo, muitos dos artigos encontrados apresentam uma abordagem mais teórica (5 artigos) e os 8 artigos de abordagem empírica, apresentam uma limitada aplicação prática, em sua maioria restritas a técnicas de observação e entrevistas, quando comparadas a variedade das técnicas utilizadas internacionalmente. Na literatura internacional, muitas técnicas de design etnográfico são adaptadas a partir das existentes, resultando em uma variedade para aplicação em diferentes contextos. Algumas variações de técnicas citadas entre os artigos internacionais foram as: cultural probes $^{1}$, magic thing ${ }^{2}$ e thing ethnography ${ }^{3}$.

Os dados acima, fazem perceber a necessidade de se entender com mais detalhes a forma de como a etnografia é abordada junto ao Design no Brasil, as técnicas utilizadas e o potencial que os autores nacionais enxergam no Design Etnográfico. A seguir, este trabalho apresentará a visão sobre esse potencial com mais detalhes.

\section{O potencial para o Design}

Compreender como as publicações encontradas interpretam as potencialidades do uso no campo do Design Antropológico pode expor a compreensão do campo e as intenções da escolha dessa abordagem estudada e/ou aplicada.

De cada artigo selecionado, foi identificado nos textos os pontos e motivos para a defesa do uso da etnografia e dois procedimentos foram feitos:

- Análise textual dos trechos selecionados, contabilizando as palavras mais frequentes e resultando em uma nuvem de palavras; e

- Interpretação textual com a síntese dos principais temas identificados.

\subsection{Nuvem de palavras}

A nuvem de palavras foi a ferramenta escolhida para síntese dos dados encontrados por fornecer aos leitores informações generalizadas sobre um conteúdo de maneira eficaz e eficiente, sem necessariamente ser preciso um envolvimento de uma leitura profunda e detalhada.

\footnotetext{
1 As "cultural probes", então e agora, são tipicamente um pacote com material, adereços e instrumentos que permitem que um informante documente o dia a dia de maneiras específicas. Diários, câmeras, mapas, sugestões de atividades, ferramentas e questionários visuais, cartões postais, etc. são frequentemente encontrados nesses pacotes.

2 A "magic thing" é uma peça em branco que é introduzida em uma situação de pesquisa do usuário. O informante deve usar este suporte para atingir seus objetivos e o estudo é feito com técnicas etnográficas.

3 A perspectiva de uma "thing" que está ativamente situada dentro das relações com outras entidades, e que tem diferentes trajetórias que os humanos, pode apresentar novos caminhos para humanos e não-humanos.
} 
http://www.revista.ufpe.br/gestaoorg

Camargo e Justo (2013) ressaltam: "a nuvem de palavras as agrupa e organiza graficamente em função da sua frequência. É uma análise lexical mais simples, porém graficamente bastante interessante, na medida em que possibilita rápida identificação das palavras-chave de um corpus".

Essa identificação das palavras, com suas frequências contabilizadas e graficamente apresentadas de modo simples, utilizando diferentes cores e tamanhos de tipografias, propiciam, para os observadores, as prioridades que foram identificadas sobre o potencial da antropologia e etnografia no campo do design.

Neste trabalho, a nuvem de palavras foi utilizada para identificar as principais palavras dentro do tema analisado e apontar pontos mais relevantes. O quadro 1 apresenta a contabilização das 20 primeiras palavras de maior frequência encontradas e a figura 1 mostra o diagrama da relação das 33 principais palavras contabilizadas.

\begin{tabular}{|l|l|l|l|}
\hline Palavra & Qt. & Palavra & Qt. \\
\hline design & 13 & compreender & 04 \\
\hline etnografia & 10 & identificar & 04 \\
\hline objetivo & 07 & requisitos & 04 \\
\hline usuário & 07 & produtos & 04 \\
\hline pesquisa & 06 & sistema & 04 \\
\hline processo & 06 & estudo & 04 \\
\hline projeto & 06 & vida & 04 \\
\hline campo & 06 & buscando & 03 \\
\hline necessidade & 05 & natureza & 03 \\
\hline desenvolvimento & 04 & inovação & 03 \\
\hline
\end{tabular}

Quadro 1 - Primeiras 20 palavras encontradas e suas contabilizações.

Fonte: elaborado pelos autores (2018).

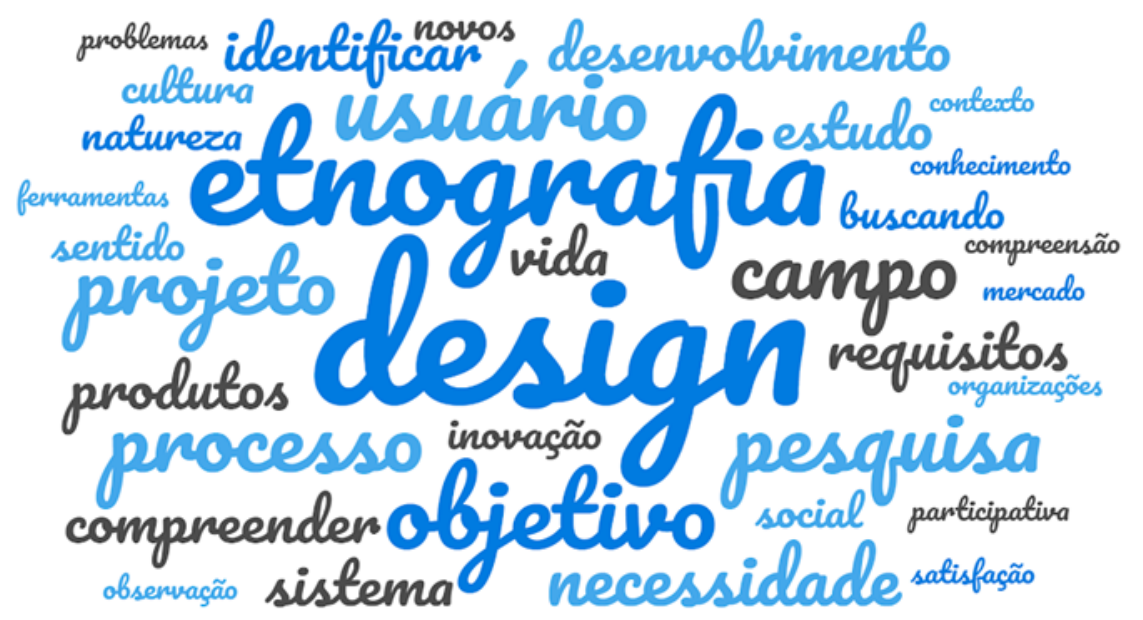

Figura 1 - Núvem de palavras.

Fonte: elaborado pelos autores (2018).

O quadro e a nuvem de palavras (Quadro 1 e Figura 1) demonstram que a palavra que mais foi destacada junto com "design" foi "etnografia" (10). Considerando diferentes abordagens, pode-se inferir que, esse resultado traz o método etnográfico como o mais utilizado/ dissertado, corroborando com os resultados de análise já identificados relacionados ao objetivo maior de imersão e compreensão do usuário.

Vale observar também as palavras "usuário, pesquisa e processo" como destaque, seguidas pela "necessidade, desenvolvimento, compreender, identificar, requisitos, produtos, sistema, estudo e vida". Apesar de ser 
interpretado e associado junto a etnografia, o design e a compreensão do contexto do usuário como caminho de potencial para o campo de design, a incidência das palavras "pesquisa e processo" evidenciam uma abordagem mais teórica dos trabalhos. Isso identifica também que, comparado aos artigos internacionais, a pesquisa brasileira encontra-se em uma fase primária do entendimento do campo, ainda o aplicando de forma tímida, evidenciado na tabela pelo menor índice de aparições das palavras "inovação, observação, participativa e satisfação".

Não são observadas uma variedade e quantidade de palavras relacionadas às técnicas e métodos com relação a etnografia e design além das palavras "observação e ferramentas". Isso remete a refletir sobre como a etnografia é vista no design e se a sua aplicação está relacionada a ferramentas e técnicas ou de fato ao método e abordagem etnográfica.

Bem no centro das contabilizações, destaca-se a palavra "necessidade", muitas vezes sendo essa uma "mediadora de águas", pois é de interesse tanto do campo teórico quanto do campo aplicado com foco no usuário a identificação das suas necessidades.

\subsection{Temas encontrados no campo do potencial para o design}

A análise textual do campo do potencial para o design trouxe também uma segunda análise e interpretação resultando em temas que englobam os pontos positivos que podem ser alcançados ao utilizar o Design Antropológico e Etnográfico nas pesquisas, teóricas e aplicadas.

\section{Imersão no contexto do usuário (ocorrência: 13 vezes);}

Em relação ao potencial da etnografia para o Design as evidências apontam como principal tema a capacidade da etnografia proporcionar imersão no contexto do usuário. Nessa imersão são mencionados temas subsequentes sobre a compreensão do usuário, suas necessidades, ideias, emoções e atividade; a otimização e usabilidade dessa imersão; a capacidade de engajamento das pessoas no Design Participativo e no fazer de futuros colaborativos. Também é enfatizada a relação dessa imersão com o processo criativo de design tanto na relação de construção de objetivos e requisitos, quanto na proposição de intervenções. Outra questão destacada é a capacidade da etnografia de proporcionar imersão dos profissionais no cotidiano do usuário;

\section{Promoção de inovação (ocorrência: 4 vezes);}

O segundo tema mais abordado envolve a promoção da inovação, mencionada quatro vezes. Esse tema envolve uma visão mais mercadológica das possibilidades da aplicação do Design Etnográfico. A perspectiva real e aplicada como uma estratégia no campo mercadológico amplamente competitivo pode viabilizar, graças a compreensão do contexto, compreender e proporcionar um reconhecimento de oportunidades oferecendo uma melhor satisfação aos usuários.

\section{Agregar valor (ocorrência: 3 vezes);}

O terceiro tema, repetido três vezes, conecta-se também à valorização dos resultados provindos do uso da abordagem etnográfica no campo do Design. Essa valorização pode ser aplicada tanto em nível de pesquisa, sobre os ricos dados adquiridos quando se imerge no contexto, quanto a possibilidade de se embutir, acrescer ou criar valor à produtos ou serviços após a compreensão os usuários em um nível aprofundado durante a fase de exploração.

\section{Visão holística - Contexto (ocorrência: 2 vezes);}

O tema visão holística aparece duas vezes como potencial para o Design, sendo abordado como a identificação dos problemas na maneira de como é usado e entendimento desse sistema englobado. Vale destacar que a visão holística, diferentemente dos resultados encontrados na literatura nacional, é um ponto forte na literatura internacional pela capacidade do método de proporcionar a compreensão situada da experiência do usuário.

\section{Etnografia no ensino de design e antropologia (ocorrência: 2 vezes);}

O penúltimo tema, sendo citado apenas duas vezes, destaca o uso da etnografia no ensino de design e antropologia. A interdisciplinaridade desses dois campos das ciências sociais, que juntas se adaptam e potencializam para diversos objetivos, e não apenas os aplicáveis, mas também no campo teórico em uma prática projetual, como modo de se desenvolver ideias alternativas sobre métodos de pesquisa.

\section{Identificação do mundano (ocorrência: 1 vez);}

Outro tema interessante, mas somente uma vez abordado, é a capacidade do Design Etnográfico de identificar os 
comportamentos mundanos do usuário. O autor descreve essa capacidade como o fato de o método revelar fatos surpreendentes.

\section{Discussão e considerações finais}

Para Papanek (1995), um designer, no sentido mais amplo do termo, é um ser humano que tenta atravessar a ponte entre a ordem e o caos, entre realizações passadas e possibilidades futuras (GUNN; OTTO; SMITH, 2013). Além disso, o design é um campo híbrido que opera a junção entre corpo e informação, entre artefato, usuário e sistema (CARDOSO, 2012, p. 237). Esses autores trazem visões que evidenciam o quão complexo o designer como profissional está inserido em sua atividade de projeção e para isso, a inserção, a visão holística e interdisciplinaridade da antropologia, podem auxiliar na possibilidade da criação de artefatos de real valor para os usuários.

Este trabalho teve como propósito mapear artigos nacionais entre 2008 e 2017 e, apesar da coleta abordar diferentes pontos de análise da compreensão de conceitos do design Etnográfico e Antropológico, assim como as ferramentas utilizadas, foi escolhido o campo de "potencial para o design" como foco para criação dessa pesquisa.

Os resultados mostram uma aproximação entre a etnografia e a antropologia junto ao design. Além disso, a pesquisa na literatura gerou algumas hipóteses, citadas a seguir, que poderão ser aprofundadas em trabalhos futuros.

A primeira hipótese é de que a literatura nacional precisa dialogar ainda mais com a literatura internacional no que se refere ao entendimento e aplicação do Design Etnográfico. É importante que haja o desenvolvimento de mais trabalhos e pesquisas no campo de Design Etnográfico que evidenciem um melhor entendimento das conceituações e, consequentemente, apresentem aplicações mais densas e propostas de variações de métodos e técnica, bem como a avaliação destes.

A partir da análise também ficou claro que o cenário nacional evidencia uma abordagem mais teórica dos trabalhos, apresentando a compreensão do campo do Design Antropológico e Etnográfico ainda em seus "primeiros passos" com aplicações que parecem, a partir das publicações, tímidas e superficiais.

A terceira hipótese se refere ao fato do campo que compreende o design e a etnografia ser considerado, para o cenário nacional, muitas vezes como apenas uma metodologia a ser aplicada e com técnicas geralmente focadas em observação e entrevista, deixando de incluir outras técnicas encontradas na literatura internacional.

Os trabalhos analisados também expressam a valorização da imersão nos contextos. No entanto, vale ressaltar que essa valorização é reconhecida na literatura nacional quando se refere a exploração de problemas e coleta de dados, utilizando o método etnográfico, em contraponto que, apesar do potencial inovativo ser identificado, aparentemente ainda é pouco explorado em sua aplicação prática.

Outra conjectura levantada se refere à visão holística da etnografia junto a identificação do que é mundano para o usuário como um ponto em potencial da área para o Design assim como é extensamente visto na literatura internacional.

E, finalmente, apesar de ainda crescente, o campo do Design brasileiro já evidencia uma compreensão do potencial da etnografia, abrindo portas para o aprofundamento dos estudos, ensinamento das técnicas e a adoção dos métodos aplicados para pesquisas futuras. Vale ressaltar também a necessidade de estudos futuros a fim de aprofundar e validar as hipóteses acima levantadas.

\section{Referências}

AGAR M. (2014). An outsider's ethnographic thoughts about design. Arts and Humanities in Higher Education, 0(0), 1-8. https://doi.org/10.1177/1474022214531478

CARDOSO, R. (2012). Design para um mundo complexo. Cosac Naify.

ALVES, C. M.; PRADO, G.; LOPES, P. G.; GOMEZ, L. S. R. (2017). Poéticas na arte, no design e na antropologia: uma análise de dois projetos artístico-fotográficos. Revista de Literatura, Linguística e Arte, p. 180-200.

ANASTASSAKIS, Z. (2013). Laboratório de Design e Antropologia: preâmbulos teóricos e práticos. Arcos Design, v. 7, n. 1, p. 178-193.

ANASTASSAKIS, Z. (2014). Design e Antropologia: considerações teóricas e experimentações práticas em diálogo com a perspectiva do antropólogo Tim Ingold. 11 $^{\circ}$ Congresso Brasileiro de Pesquisa e 


\section{Desenvolvimento em Design.}

ARAUJO, E. P. (2012). Um estudo sobre Etnografia aplicada ao Design. Dissertação de Mestrado, PUC-Rio, p. $1-104$.

BORMAN, K. M.; LECOMPTE, M. D.; GOETZ, J. P. (1986). Ethnographic and qualitative research design and why it doesn't work. American Behavioral Scientist, v. 30, n. 1, p. 42-57.

CAMARGO, B. V.; JUSTO, A. M. (2013). Iramuteq: Um Software Gratuito para Análise de Dados Textuais. Periódico Eletrônico de Psicologia, vol. 21, no. 2, Ribeirão Preto, Dez. Disponível em: http://pepsic.bvsalud.org/pdf/tp/v21n2/v21n2a16.pdf

DROSTE, M. (2014). Bauhaus, 1919-1933. Editora: Taschen, p. 240.

SOUZA FIALHO, U. F. C. (2014). A pesquisa com usuários no processo de design: sugestões a partir de uma abordagem etnográfica. Dissertação de Mestrado, Programa de Pós-Graduação em Design, UFRGS. Disponível em: <http://www.lume.ufrgs.br/handle/10183/127786>

DIAS FILHO, C. S. (2007). Design Numa Perspectiva Cultural. III ENECULT - Encontro de Estudos Multidisciplinares em Cultura, 2007.

GIACCARDI, E.; CILA, N.; SPEED, C.; CALDWELlL, M. (2016). Thing Ethnography. Proceedings of the 2016 ACM Conference on Designing Interactive Systems - DIS '16, 377-387. 2016. https://doi.org/10.1145/2901790.2901905.

GUNN, W.; OTTO, T.; SMITH, R. C. (Ed.). (2013). Design anthropology: theory and practice. A\&C Black.

IBARRA, M. C.; ANASTASSAKIS, Z. (2016). O design e suas possíveis interações com práticas criativas desenvolvidas por não-designers. Arcos Design, p. 165-176, 2016.

KAARST-BROWN, M. L.; GUZMAN, I. R. (2014). Cultural richness versus cultural large scale insights. Proceedings of the 52 ${ }^{\text {nd }} \mathrm{ACM}$ Conference on Computers and People Research - SIGSIM-CPR '14, 5558. https://doi.org/10.1145/2599990.2600002

LUCCA, A. S. (2016). A etnografia rápida no metaprojeto de design para o território. Logo e-Revista, ISSN: 22382542, p. 23-36.

WATANABE, W. M.; FORTES, R. P. M.; (2012). Revisão Sistemática sobre princípios de design de aplicações web acessíveis para analfabetos funcionais. World Wide Web Internet And Web Information Systems, p. 403-417.

MEYER, G.; DAMAZIO, V. (2012). Notas de uma etnografia do Design. Strategic Design Research Journal, v. 5, n. 3, p. 120-128, Disponível em: <http://revistas.unisinos.br/index.php/sdrj/article/view/sdrj.2012.53.04>.

MOTA, C.; SIRITO, M. (2016). Design em movimento: repensando o design através do encontro com as ciências sociais. [S.d.].

NERY, C. G.; MAGALHÃES, L. A. C.; CÂMARA, J. J. D.; LANA, S. L. B. (2016). Os Livros Digitais de Fotografia da Coleção Festejo Maior: nexos possíveis entre design, etnografia e antropologia visual, In: Anais do $12^{\circ}$ Congresso Brasileiro de Pesquisa e Desenvolvimento em Design.

PERPÉTUO, N. C. F.; NORONHA, R. G. (2016). Estar lá, mas também do outro lado da tela: limites e possibilidades da etnografia em ambientes virtuais e a percepção de blogueiras de moda sobre design, produtos e sustentabilidade. $1^{\circ}$ Congresso Brasileiro de Pesquisa e Desenvolvimento em Design.

PINK, S. (2014). Digital-visual-sensory-design anthropology: Ethnography, imagination and intervention. Arts and Humanities in Higher Education, 13(4), 412-427. 2014. https://doi.org/10.1177/1474022214542353

PINK, S.; FORS, V.; GLÖSS, M. (2017). Automated futures and the mobile present: In-car video ethnographies. Ethnography, 146613811773562. https://doi.org/10.1177/1466138117735621

RODE, J. A. (2011). Reflexivity in Digital Anthropology. CHI '11 Proceedings of the SIGCHI Conference on Human Factors in Computing Systems, 1-10. 2011 https://doi.org/10.1145/1978942.1978961

ROSA, J. G. S.; GURGEL, A.; PASSOS, M. (2012). Técnicas Baseadas em Etnografia e Prototipagem no Design de interface de Aplicativo Mobile para Gerenciamento Acadêmico. InfoDesign, v. 9, n. 2, p. 88-99, 2012. Disponível em: <http://www.infodesign.org.br/revista/index.php/infodesign/article/viewFile/123/94> 
SEGELSTROM, F.; HOLMLID, S. (2015). Ethnography by design: On goals and mediating artefacts. Arts and Humanities in Higher Education, 14(2), 134-149. 2015. https://doi.org/10.1177/1474022214560159

SMITH, R. C.; KJÆRSGAARD, M. G. (2014). Design anthropology in participatory design from ethnography to anthropological critique?, Proceedings of the $13^{\text {th }}$ Participatory Design Conference on Short Papers, Industry Cases, Workshop Descriptions, Doctoral Consortium Papers, and Keynote Abstracts - PDC '14 Volume 2, 217-218. 2014. https://doi.org/10.1145/2662155.2662209.

STAPPERS, P. J.; VAN RIJN, H.; KISTEMAKER, S. C.; HENNINK, A. E.; SLEESWIJK V. F. (2009). Designing for other people's strengths and motivations: Three cases using context, visions, and experiential prototypes. Advanced Engineering Informatics, 23(2), 174-183. Disponível em: https://doi.org/10.1016/j.aei.2008.10.008

TARACHUCKY, L.; GOMEZ, L. S. R. (2013). Pesquisa Etnográfica Aplicada a Processos de Inovação, Estado da Arte e Perspectivas. p. 2-8.

TAVARES, P.; CALDAS, L. C. A.; FERREIRA, S. B. L. (2010). Observações Etnográficas na Avaliação da Usabilidade de Dispositivos Móveis de Coleta de Dados Estatísticos. Conferência Interaction South America, Universidade Positivo, Curitiba, Disponível em: $<$ http://www.agner.com.br/download/artigos/Artigo - Congresso Interaction SA 2010.pdf $>$.

TOÉ, R. D. A. D.; BELO, J. A.; TOSTA, H. (2011). A pesquisa etnográfica como estratégia para inovação. Revista E-Tech: Tecnologias para Competitividade Industrial - ISSN - 1983-1838, v. 4, n. 1, p. 68-81, Disponível em: <http://revista.ctai.senai.br/index.php/edicao01/article/view/176>. 